\title{
Applications of the Wireless LAN Technology in The Nursing Work
}

\author{
Junlei Zhang ${ }^{1, a}$, Huimin Zhang ${ }^{2, b}$, Cuimin Lian $^{3, \mathrm{c}}$, Rong LI ${ }^{* 2, \mathrm{~d}}$
}

${ }^{1}$ Children and adolescents psychiatric of Second Affiliated Hospital of Xinxiang Medical University, Xinxiang, Henan Province, China

${ }^{2}$ Nursing college of Xinxiang Medical University, Xinxiang, Henan Province, China,

${ }^{3}$ Neurological in-patients of Second Affiliated Hospital of Xinxiang Medical University, Xinxiang, Henan Province, China

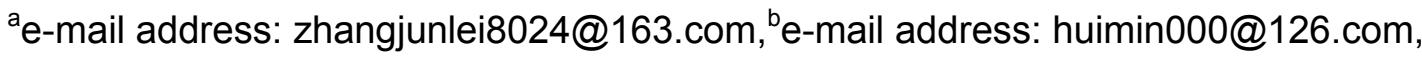

ce-mail address: 714762051@qq.com, ${ }^{d}$ e-mail address: Ir2665@yahoo.com.cn

Keywords: Wireless LAN Technology; Care; Application

\begin{abstract}
With the rapid development of computer network technology, wireless networks have developed to wireless wide area network from a single wireless personal area network. Expansion of coverage was spread from a few meters to a global range and transmission speed was also doubled. The wireless network is gradually used into complicated module network environment, especially in the field of medical treatment, depending on the flexibility and the sound extendibility of its module network. This article is to introduce the wireless LAN technique and its character, and also to express its scientific use in the care.
\end{abstract}

\section{Introduction}

With the rapid development of the computer network technology, the wireless network technology was widely used in the many industries, especially in the field of medical treatment. Wireless LAN effective overcome the shortcomings of the wired network, it's gradually used in many areas, such as going the rounds of the wards, nursing by the bed, calling and communication, nursing and monitoring, administration and delivering of medicine. At present, to further improve efficiency and improve health care quality, influential hospital at home and abroad have adopted wireless LAN-based wireless network[1].

\section{Wireless LAN Technology}

Wireless LAN is a computer network technology combined with wireless communication products, it can be achieved without wiring between the computer and other network devices interconnected network. It uses radio frequency technology to replace the old coaxial cable and twisted wire form a computer network. It can provide all of the features which are offered by traditional wired LAN. Wireless LAN technology has been improved with such increasing demand on the network communication rate. IEEE International standards group launched: IEEE802 11, EEE802 11b, IEEE802 11a, IEEE802 11g standard, etc[2], and specially formulated IEEE802 11 i standards for the safety of wireless LAN. It provides a reliable security guarantee for wireless LAN data transmission. Wireless LAN has the following advantages.

Easy Installation. We can avoid or reduce the workload of network cabling using wireless LAN , generally it can cover the entire area of the local area network as long as we instal one or more the access point.

Strong Mobility. One of the biggest advantages of wireless network is mobility. Users can mobile work within the area covered by the wireless LAN. Medical staff can operate system for mobile work in any location within the network, such as the ward, operating room, treatment room, nursing unit [3].

Flexible Networking. Wireless local area network can be composed of a variety of topologies, and can be very easily from point to point mode expansion a small number of users to the infrastructure network of thousands of users. 
Cost Advantage. Wireless LAN to avoid or reduce the office location or network topology changes and network building to happen again, thus saving a lot of user fees. We can get more rewarding investment in the wireless LAN in the frequently move and dynamic environment[4].

\section{The New Features of Care}

With high-quality care activities, the model of care are changing. With high-quality care activities, the model of care are changing. Nurses are not only responsible for the basic care, but also carry out special needs services, family services. As much as possible to meet the needs of the patient's various medical information, but also continuously improve the quality of care and safety management, this requires improvement of medical services by time, space, living facilities, care and treatment services, the environment and strengthening a dominant position to highlight its human management. With setting up the wireless LAN in the unit, nurse can rapid gain the information about the patient, medical history, vital signs, test results and other information without the limitation by the traditional nursing station. As effectively as possible communication with the patient, so that patients can get high efficiency and quality nursing.

\section{Applications of the Wireless LAN Technology in the Nursing Work}

Based on the advantages of the wireless LAN technology, It has been widely applied in the hospital clinical management system. At present, the domestic hospital in nursing is mainly used in the following respects.

Outpatient Service Mobile Infusion System. The system realized respectively that the nurse check accurately to patients identity and drug, and help patients in the correct time, accurate location in time mainly by the use of the bar code technology, wireless network technology and mobile computer technology. The system changes the former tedious check the link, ensures the accuracy and safety of the infusion processes.

Mobile Nurse Workstation. Mobile nurse workstations to the hospital information system for support platform to PDA and tablet computer for hardware platform, the wireless local area network for network platform, and realize the doctor's advice to the execution of the follow-up, to ensure the data collection, nursing care task management and nursing of real-time monitoring, improve work efficiency, optimize the nursing process, greatly promote the hospital information system and the development of digital trend[5].

Call Communication. Forming the wireless local area network, the hospital can use voip system in place of the traditional communication equipment, and can transmit voice and data in the same network, and provide two-way voice communication. Medical personnel can through hand-held devices receiving the patient's call, directly speak to patients and understanding the patient's needs from the system.

Care Monitoring. After forming the wireless local area network, it can be equipped with wireless camera, through the network configuration parameters, it can be used for monitoring ward, pharmacy, and other important places. It can further improve medical staff's working efficiency if Wireless camera combined with the paging system. Staff can directly monitor to the patient's condition after paging signal through networked computer terminal, and adopt corresponding measures of medical treatment, this has an important role for the critically ill patient[6].

The wireless LAN is expanding on the application in health care with the continuous improvement of the wireless LAN and the increased demand of the hospital management information system. The wireless LAN will play a more and more important role in improving work deficiency, promoting the nursing service quality by the applications in the medical care. 


\section{Acknowledgment}

This paper is a teaching reform task supported by Association of Social Sciences, which is the subject of Top three hospitals in the undergraduate nursing students needs and abilities and quality requirements (SKL-2011-2317). Rong $\mathrm{Li}$ is corresponding author.

\section{References}

[1] Wang J, Du H. Setting up a Wireless Local Area Network (WLAN) for a healthcare system[J]. Int J Electron Healthc. 2005,1(3):335-48.

[2] Calcagnini G, Mattei E, Censi F, et al. Electromagnetic compatibility of WLAN adapters with life-supporting medical devices[J]. Health Phys. 2011 May;100(5):497-501.

[3] Kang K, Park KJ, Song JJ, et al. A medical-grade wireless architecture for remote electrocardiography[J]. IEEE Trans Inf Technol Biomed. 2011,15(2):260-7.

[4] Cha YD, Yoon G. Ubiquitous health monitoring system for multiple users using a ZigBee and WLAN dual-network[J]. Telemed J E Health. 2009,15(9):891-7.

[5] Sawa T, Funahara T, Nagatani H, et al. WLAN PDA to improve efficiency in patient care documentation[J]. AMIA Annu Symp Proc. 2005:1107

[6] Varshney U. Patient monitoring using infrastructure-oriented wireless LANs[J]. Int J Electron Healthc. 2006, 2(2):149-63. 